\title{
Atitudes alimentares e insatisfação corporal em estudantes de nutrição de uma instituição particular de ensino superior, matriculados em diferentes períodos do curso
}

Food attitudes and body dissatisfaction in nutrition students from a private higher education institution enrolled in different course periods

Actitudes alimentarias e insatisfacción corporal en estudiantes de nutrición de una institución de educación superior privada, matriculados en diferentes períodos del curso

Recebido: 12/10/2021 | Revisado: 20/10/2021 | Aceito: 29/10/2021 | Publicado: 01/11/2021

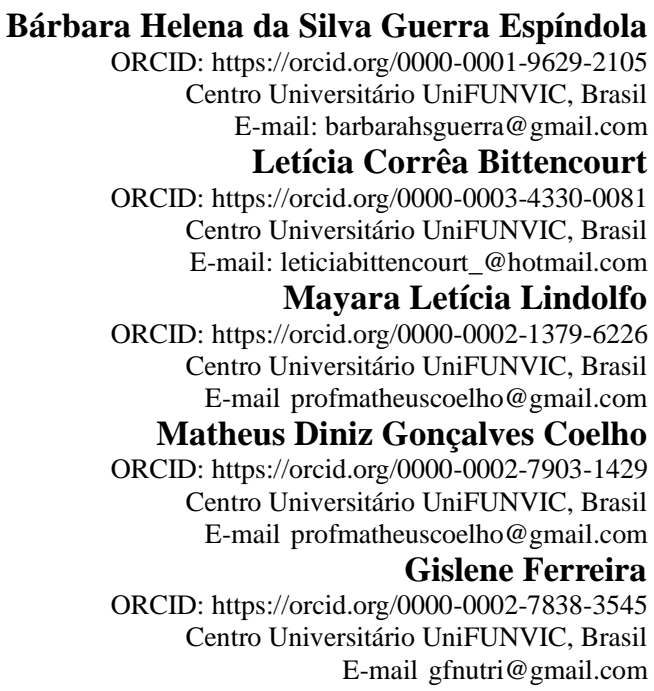

\section{Resumo}

O comportamento alimentar sofre influência de diversos fatores, sejam psicológicos e emocionais, ou socioeconômicos, onde o estilo de vida norteia a alimentação. $\mathrm{O}$ ingresso na vida acadêmica universitária aflora ainda mais essas emoções, devido à pressão para a adequação do corpo dentro dos padrões impostos, somado às expectativas de um bom desempenho profissional, principalmente em estudantes da área da nutrição. O objetivo deste trabalho foi analisar mudança comportamental dos acadêmicos de nutrição com o decorrer do curso, em relação aos hábitos alimentares e estilo de vida. Foram avaliados 37 estudantes de nutrição, de uma instituição privada, na cidade de Pindamonhangaba - São Paulo, matriculados em diferentes períodos. Foram aplicados os questionários Eating Attitudes Test - EAT-26 e Body Shape Questionnaire - BSQ, para análise de transtornos alimentares e distorção da imagem corporal, respectivamente, bem como, um questionário de frequência de consumo alimentar e outro contendo questões pessoais, sobre o perfil social e estilo de vida. Fez-se análise descritiva e estatística dos resultados, para verificar associação da mudança comportamental dos hábitos alimentares e estilo de vida dos acadêmicos de nutrição com o decorrer do curso. Apesar de encontrar transtorno alimentar leve e distorção da imagem corporal em todos os períodos, não houve diferença significativa entre as turmas avaliadas e nem presença significativa destes transtornos no grupo. No entanto, os hábitos alimentares, e consequentemente, o estado nutricional da maioria destes universitários se encontram adequados, fato que pode ter sido influenciado pela aquisição de novos conhecimentos na área. Palavras-chave: Transtorno da compulsão alimentar; Comportamento alimentar; Imagem corporal; Universitários.

\footnotetext{
Abstract

Eating behavior is influenced by several factors, such as psychological, emotional, or socioeconomic, it means that lifestyle guides eating. The initiation into academic life makes these feelings wider, due to stress to fitthe body in the imposed standards, it added to the expectations of a good professional performance, especially about students of Nutrition area. The objective of this research was to analyze behavioral changes of Nutrition students during the course, linked to eating habits and lifestyle. 37 nutrition students from a private institution in Pindamonhangaba - SP, who were enrolled in different periods, were evaluated. The questionnaires Eating Attitudes Test (EAT-26) and Body Shape Questionnaire (BSQ) were applied to analyze
} 
eating disorders and body image disturbance, respectively, as well as a Food Frequency Questionnaire and another one with personal questions about social profile and lifestyle. Descriptive and statistical analysis of the results was carried out to verify the association between the behavioral change of eating habits and lifestyle of nutrition students during the course. Although results of mild eating disorders and body image dissatisfaction were found in all periods, there was no significant difference between the classes assessed and no significant presence of these disorders in the group. However, the eating habits, and consequently, the nutritional status of most of these students, are adequate, a fact that may have been influenced by the acquisition of new knowledge in the area.

Keywords: Binge-eating disorder; Feeding behavior; Body image; College students.

\section{Resumen}

La conducta alimentaria está influenciada por variosfactores, yasean psicológicos y emocionales o socioeconómicos, donde el estilo de vida guía la alimentación. El ingreso a la vida académica universitaria toca aún más estas emociones, debido a la presión por adecuar el cuerpo dentro de los estándares impuestos, sumada a las expectativas de un buen desempeño profesional, especialmente entre los estudiantes del campo de la nutrición. El objetivo de este trabajo fue analizar el cambio de comportamiento de los estudiantes de nutrición durante el curso, en relación con los hábitos alimentarios y el estilo de vida. Fueron evaluados 37 estudiantes de nutrición de una institución privada de la ciudad de Pindamonhangaba - São Paulo, matriculados en diferentes períodos. Se aplicaron los cuestionarios Eating Attitudes Test - EAT-26 y Body Shape Questionnaire - BSQ para analizar los trastornos alimentarios y la distorsión de la imagen corporal, respectivamente, así como un cuestionario de frecuencia de consumo de alimentos y otro con preguntas personales sobre el perfil social y estilo de vida. Se realizó un análisis descriptivo y estadístico de los resultados para verificar la asociación del cambio conductual en los hábitos alimentarios y el estilo de vida de los estudiantes de nutrición con el transcurso del curso. Apesar de encontrar transtornos alimentarios leves y distorsión de la imagen corporal en todos los períodos, no hubo diferencia significativa entre los grupos evaluados ni presencia significativa de estos trastornos en el grupo. Sin embargo, los hábitos alimentarios y, en consecuencia, el estado nutricional de la mayoría de estos universitarios son adecuados, hecho que puede haber sido influenciado por la adquisición de nuevos conocimientos en el área.

Palabras clave: Transtornos compulsón alimentary; Comportamiento alimentario; Imagem corporal; Estudiantes universitarios.

\section{Introdução}

O comportamento alimentar é algo complexo, onde o estilo de vida norteia a alimentação, como o local, horários, número de companhias nas refeições e condições socioeconômicas (Pereira, Da Silva \& De Sá, 2015; Dantas \& Silva, 2019). As informações nutricionais provocam mudanças na escolha dos alimentos e, dependendo da trajetória do indivíduo e do seu curso de vida, estas mudanças poderão ou não ser convenientes. O ingresso na vida acadêmica universitária aflora ainda mais essas emoções. Segundo Leitão et al. (2015), fatores psicológicos que influenciam no comportamento alimentar, como depressão, ansiedade e sentimentos inespecíficos de tensão são identificados como ativadores da compulsão alimentar. Indivíduos com esse padrão alimentar apresentam, além da ansiedade e depressão, mais frequentemente sentimentos de autodesprezo, repulsa a sua forma corporal, preocupação somática, estresse e prejuízo em suas relações interpessoais.

Além disso, a pressão para a adequação do corpo dentro dos padrões impostos, somado às expectativas de um bom desempenho profissional, podem favorecer o desenvolvimento de Transtornos Alimentares (Karakus et al., 2017). É crescente o número de jovens que têm vivenciado algum tipo de transtorno alimentar nos últimos anos, cujos fatores são muitos, quer seja pela distorção da imagem corporal, ou por pressão das pessoas com as quais convive, ou até mesmo por questões emocionais e psicológicas tão intrínsecas, que o próprio jovem ou adolescente desconhece. Alguns autores indicam que o risco de perturbações do comportamento alimentar ou até mesmo a sua prevalência, é superior nos estudantes da área da nutrição e dietética, comparativamente aos estudantes de outros cursos (Yu \& Tan, 2016; Oliveira et al., 2017; Agopyan et al., 2018).

Dessa forma, torna-se interessante conhecer como os universitários de uma determinada área da saúde, como a nutrição, conciliaria as informações que adquire ao longo do curso com sua vida extra acadêmica. Fato é que a disponibilidade de conceitos e informações acerca de saúde, qualidade de vida e alimentação são vultosas e isto pode influenciar na vida do universitário, causando algum impacto certamente. Diante do exposto, este trabalho tem como objetivo, analisar a mudança comportamental dos acadêmicos de nutrição com o decorrer do curso, em relação aos hábitos alimentares e estilo de vida; uma vez que passam a adquirir informações sobre como ter uma vida mais saudável, mas também passam a ser mais cobrados em 
relação à estética corporal.

\section{Metodologia}

Trata-se de uma pesquisa de campo, de base populacional, com coleta de dados primários de forma descritiva, quantitativa, de caráter transversal e observacional realizada com um grupo de estudantes matriculados em todos os períodos vigentes no ano de 2020 ( $2^{\circ}$ ao $8^{\circ}$ períodos) do Curso de Nutrição, do Centro Universitário UniFUNVIC em Pindamonhangaba-SP, Brasil, durante os meses de maio a novembro de 2020.

Para cálculo do tamanho da amostra mínima significativa, considerou-se que no curso de nutrição noturno do UniFUNVIC, no ano de 2020, a média de alunos matriculados por turma era de cerca de 18 alunos e que esse número tendia a diminuir nos últimos semestres do curso. Assim, optou-se em trabalhar com um mínimo de 10 alunos por turma, considerando ainda as recusas em participar da pesquisa e desistência do curso no decorrer da coleta de dados. Dessa forma, a amostra mínima significativa total calculada foi de 40 acadêmicos, sendo 10 de cada ano.

Foram incluídos na pesquisa alunos de ambos os sexos, maiores de 18 anos, matriculados no curso de Nutrição do UniFUNVIC do primeiro, segundo, terceiro e quarto anos e excluídos alunos de outros cursos da Instituição, que não o de nutrição; com idade inferior a dezoito anos, aqueles que se recusarem a participar.

O estudo foi iniciado após aprovação do Comitê de Ética em Pesquisa da Faculdade de Pindamonhangaba sob o parecer n³.413.200 em 25/06/2019 e após leitura e assinatura do Termo de Consentimento Livre e Esclarecido.

Para a coleta de dados foram utilizados quatro questionários estruturados, autoaplicáveis, contendo perguntas de múltipla escolha. O primeiro continha perguntas envolvendo questões pessoais, sobre o perfil social e estilo de vida, tais como, gênero, faixa etária, se pratica atividade física, como classifica seu nível atual de saúde, como considera seu nível de estresse e ansiedade, se está satisfeito com seu peso, peso atual e altura referidos pelo próprio participante da pesquisa, para cálculo posterior de IMC. O segundo questionário buscou conhecer o consumo alimentar do indivíduo, por meio do questionário de frequência alimentar adaptado de Henn et al., (2010) contendo alimentos saudáveis, como frutas, legumes e verduras.

Os participantes da pesquisa também foram avaliados quanto às atitudes alimentares e satisfação com sua imagem corporal, utilizando os questionários autoaplicáveis, o Eating Attitudes Test - EAT- 26 e o Body Shape Questionnaire - BSQ, respectivamente. Tais instrumentos são de fácil aplicação, eficientes e econômicos na avaliação de grande número de indivíduos, além de permitirem aos entrevistados revelar um comportamento que, por considerarem vergonhoso, poderia deixá-los relutantes numa entrevista face-a-face (Nascimento et al., 2020).

O Eating Attitudes Test (EAT) ou Teste de Atitudes Alimentares é um instrumento de auto aplicação com 26 questões, avaliados em escala "Likert" com seis opções gradativas de resposta que são pontuadas (3 - sempre, 2 - muitas vezes, 1 - às vezes, 0-poucas vezes, quase nunca e nunca) (Garner et al., 1982). A única questão que apresenta pontos em ordem invertida é a 25 , sendo que para respostas mais sintomáticas, como o sempre, muitas vezes e às vezes, não são dados pontos, e para as alternativas poucas vezes, quase nunca e nunca, são conferidos 1, 2 e 3 pontos, respectivamente. O ponto de corte estabelecido é de 21 pontos. O teste deve ser usado como índice da gravidade de preocupações típicas de pacientes com transtorno alimentar, particularmente intenção de emagrecer e medo de ganhar peso. Pode ser utilizado para avaliar presença de sintomas de transtorno alimentar em populações não clínicas, mas não deve ser instrumento de diagnóstico (Fortes et al., 2016). A primeira versão do EAT desenvolvida por Garner \& Garfinkel (1979) era composta por 40 itens, e, em função do tempo gasto em seu preenchimento, uma versão abreviada foi proposta em seguida por Garner, Olmsted, Bohr, \& Garfinkel, (1982), contendo 26 itens, sendo a versão utilizada no presente trabalho.

O Body Shape Questionnaire (BSQ) é usado para avaliar o grau de insatisfação com a imagem corporal real e/ou imaginária e mede as preocupações com a forma do corpo, autodepreciação e o sentimento de "estar gordo (a)". É composto 
por 34 questões de autopreenchimento, cujas respostas obtidas recebem uma pontuação ( 1 - nunca; 2 - raramente; 3 - às vezes; 4 - frequentemente; 5 - muito frequentemente; 6 - sempre) e o somatório das mesmas varia entre 34 a 204 pontos. Assim, quanto maior a pontuação, maior a distorção da imagem corporal. Valores menores que 80 classificam a ausência de distorção; entre 80 a 110, distorção leve; 111 a 140, distorção moderada e se a pontuação for maior que 140, distorção de imagem corporal grave (Cooper et al., 1987).

Devido à pandemia de Covid-19 que ocasionou a suspensão das aulas presenciais exatamente na época da coleta de dados, todos os questionários, juntamente com o Termo de Consentimento Livre e Esclarecido, foram enviados através de um link para o e-mail institucional dos alunos do curso de Nutrição e desta forma, aplicados pela plataforma Google forms, no formato online.

Os dados obtidos foram tabulados no programa Excel e a análise estatística para avaliar diferenças significativas entre diversas variáveis (ocorrência e tipo de transtorno, ocorrência e intensidade dos transtornos em relação ao nível de progresso dos entrevistados no curso, ocorrência e intensidade de desconforto com a aparência em relação ao nível de progresso dos entrevistados no curso, diferença dos hábitos alimentares entre os alunos matriculados nos diferentes períodos do curso) foi realizada utilizando-se o programa Bioestat 5.0, como ferramenta de apoio. Os testes aplicados foram: os testes G, com nível de significância $<0,05$; ANOVA/Tukey, com $\mathrm{p}<0,05$ e Kruskal Wallis/Dunn $\mathrm{p}<0,05$, para verificar associação da mudança comportamental dos acadêmicos de nutrição com o decorrer do curso, em relação aos hábitos alimentares, estilo de vida e presença de transtornos alimentares.

\section{Resultados}

\subsection{Caracterização da amostra}

Foram avaliados 37 alunos matriculados do $1^{\circ}$ ao $4^{\circ}$ anos ( $2^{\circ}$ ao $8^{\circ}$ períodos) do Curso de Nutrição, do Centro Universitário UniFUNVIC em Pindamonhangaba/SP, Brasil, sendo 83,78\% ( $\mathrm{n}=31$ ) do sexo feminino e 16,21\% ( $\mathrm{n}=6$ ), do sexo masculino.

Dentre os alunos avaliados, 7 alunos $(18,91 \%)$ eram do $2^{\circ}$ período, 6 alunos $(16,21 \%)$ do $4^{\circ}$ período, $11(29,72 \%)$, do $6^{\circ}$ período e $13(35,13 \%)$, do $8^{\circ}$ período.

A faixa etária predominante foi de 18 a 25 anos $(72,97 \%$; $n=27)$, seguida por 26 a 33 anos $(16,21 \%$; $=6)$ e 34 a 41 anos $(5,40 \% ; n=2)$. Apenas um aluno $(2,70 \%)$ se encontrava na faixa etária entre 42 e 49 anos e um aluno tinha 50 anos ou mais.

Quanto à prática de atividade física, os alunos matriculados no primeiro ano do curso foram os que relataram menor frequência de atividade física, com apenas 3 alunos (42,90\%) afirmando praticar exercícios físicos regularmente, enquanto 8 alunos do $4^{\circ}$ ano $(61,50 \%)$ relataram praticar atividade física com frequência. Os resultados estão apresentados no Quadro1.

Quadro 1 - Prática de atividade física entre os 37 alunos do curso de Nutrição do Centro Universitário UnifUNVIC. Pindamonhangaba/SP, Brasil. 2020. Distribuição por turma.

\begin{tabular}{|l|c|c|}
\hline Prática de Atividade Física & Praticam & Não Praticam \\
\hline $1^{\circ}$ ANO & $42,90 \%(n=3)$ & $57,10 \%(n=4)$ \\
\hline $2^{\circ}$ ANO & $50,00 \%(n=3)$ & $50,00 \%(n=3)$ \\
\hline $3^{\circ}$ ANO & $54,50 \%(n=6)$ & $45,50 \%(n=5)$ \\
\hline $4^{\circ}$ ANO & $61,50 \%(n=8)$ & $38,50 \%(n=5)$ \\
\hline
\end{tabular}

Fonte: Dados da pesquisa (2021). 
Em relação ao estado geral de saúde, os alunos que classificaram sua saúde como excelente, foram em sua maioria os matriculados no $3^{\circ}$ ano $(27,30 \%$; $n=3)$, os que classificaram como boa, foram principalmente, os alunos do $4^{\circ}$ ano $(69,20 \%$; n=9) e os que, com maior frequência disseram considerar seu estado de saúde como regular, foram os do $2^{\circ}$ ano $(33,30 \%$; $\mathrm{n}=2$ ), conforme demonstrado no Quadro 2. Nenhum aluno considerou possuir saúde ruim ou péssima.

Quadro 2 - Classificação da saúde segundo os alunos do curso de Nutrição do Centro Universitário UniFUNVIC. Pindamonhangaba/SP, Brasil. 2020. Distribuição por turma.

\begin{tabular}{|l|c|c|c|c|}
\hline $\begin{array}{l}\text { Como consideram seu } \\
\text { estado geral de saúde }\end{array}$ & $\begin{array}{c}\text { Saúde } \\
\text { Excelente }\end{array}$ & Saúde Boa & $\begin{array}{c}\text { Saúde } \\
\text { Regular }\end{array}$ & $\begin{array}{c}\text { Saúde Ruim/ } \\
\text { Péssima }\end{array}$ \\
\hline $1^{\circ}$ ANO & $14,3 \%(n=1)$ & $71,4 \%(n=5)$ & $14,3 \%(n=1)$ & 0 \\
\hline $2^{\circ}$ ANO & 0 & $66,7 \%(n=4)$ & $33,3 \%(n=2)$ & 0 \\
\hline $3^{\circ}$ ANO & $27,3 \%(n=3)$ & $63,6 \%(n=7)$ & $9,1 \%(n=1)$ & 0 \\
\hline $4^{\circ}$ ANO & $15,4 \%(n=2)$ & $69,2 \%(n=9)$ & $15,4 \%(n=2)$ & 0 \\
\hline
\end{tabular}

Fonte: Dados da pesquisa (2021).

Quanto à presença de ansiedade e estresse, os alunos do $4^{\circ}$ ano foram os que mais relataram apresentar sintomas relacionados à ansiedade e estresse $\left(76,90 \% \mathrm{n}=10 \mathrm{em}\right.$ ambos sintomas); em contraponto aos alunos do $1^{\circ}$ ano, cuja maioria negou sintomas de estresse e ansiedade, sendo que apenas 2 alunos (28,60\%) afirmaram a presença de ansiedade e $3(42,90 \%)$, de estresse, frequentemente. Os resultados encontrados estão descritos nos Quadro 3.

Quadro 3 - Relato de ansiedade e estresse dos alunos do curso de Nutrição do Centro Universitário UniFUNVIC. Pindamonhangaba/SP, Brasil. 2020. Distribuição por turma.

\begin{tabular}{|l|c|c|}
\hline Ansiedade & Sim & Não \\
\hline $1^{\circ}$ ANO & $28,60 \%(n=2)$ & $71,40 \%(n=5)$ \\
\hline $2^{\circ}$ ANO & $83,30 \%(n=5)$ & $16,70 \%(n=1)$ \\
\hline $3^{\circ}$ ANO & $81,80 \%(n=9)$ & $18,20 \%(n=2)$ \\
\hline $4^{\text {o }}$ ANO & $76,90 \%(n=10)$ & $23,10 \%(n=3)$ \\
\hline Estresse & Sim & Não \\
\hline $1^{\circ}$ ANO & $42,90 \%(n=3)$ & $57,10 \%(n=4)$ \\
\hline $2^{\circ}$ ANO & $66,70 \%(n=4)$ & $33,30 \%(n=2)$ \\
\hline $3^{\circ}$ ANO & $63,60 \%(n=7)$ & $36,40 \%(n=4)$ \\
\hline $4^{\circ}$ ANO & $76,90 \%(n=10)$ & $23,10 \%(n=3)$ \\
\hline
\end{tabular}

Fonte: Dados da pesquisa (2021).

Quando questionados sobre estarem satisfeitos com seu peso corporal, a satisfação com o peso atual predominou nos alunos do $3^{\circ}$ ano $(63,60 \%$; $n=7)$, enquanto $100 \%(n=6)$ dos alunos do $2^{\circ}$ ano disseram não estar satisfeitos com seu peso. (Quadro 4) 
Quadro 4 - Satisfação ou insatisfação no peso corporal dos alunos do curso de Nutrição do Centro Universitário UniFUNVIC. Pindamonhangaba/SP, Brasil. 2020. Distribuição por turma.

\begin{tabular}{|l|c|c|c|}
\hline Satisfeito com o peso & Sim & $\begin{array}{c}\text { Não, gostaria de } \\
\text { diminuir }\end{array}$ & $\begin{array}{c}\text { Não, gostaria de } \\
\text { aumentar }\end{array}$ \\
\hline $1^{\circ}$ ANO & $28,60 \%(n=2)$ & 0 & $71,40 \%(n=5)$ \\
\hline $2^{\circ}$ ANO & 0 & $50,00 \%(n=3)$ & $50,00 \%(n=3)$ \\
\hline $3^{\circ}$ ANO & $63,60 \%(n=7)$ & $27,30 \%(n=3)$ & $9,10 \%(n=1)$ \\
\hline $4^{\circ}$ ANO & $38,50 \%(n=5)$ & $46,20 \%(n=6)$ & $15,40 \%(n=2)$ \\
\hline
\end{tabular}

Fonte: Dados da pesquisa (2021).

Com relação ao IMC mensurado com os dados de peso e altura relatados pelos próprios participantes da pesquisa, apesar do baixo peso estar presente apenas no $1^{\circ}$ ano e o sobrepeso e obesidade ser mais prevalente no $4^{\circ}$ ano, não houve diferença significativa entre as turmas avaliadas (teste $\mathrm{G}$, com significância $<0,05$ ), conforme observado no Quadro 5.

Quadro 5 - IMC de alunos do curso de Nutrição do Centro Universitário FUNVIC. Pindamonhangaba/SP, Brasil. 2020.

\begin{tabular}{|l|l|l|l|l|}
\hline IMC $*$ & Baixo Peso & Eutrófico & Sobrepeso & Obesidade \\
\hline $1^{\circ}$ ANO & $42.90 \%(n=3)$ & $57.10 \%(n=4)$ & 0 & 0 \\
\hline $2^{\circ}$ ANO & 0 & $83.34 \%(n=5)$ & $16.66 \%(n=1)$ & 0 \\
\hline $3^{\circ}$ ANO & 0 & $63.60 \%(n=7)$ & $27.30 \%(n=3)$ & $9.10 \%(n=1)$ \\
\hline $4^{\circ}$ ANO & 0 & $53.84 \%(n=7)$ & $30.76 \%(n=4)$ & $15.38 \%(n=2)$ \\
\hline
\end{tabular}

*Ausência de diferença significativa (Teste G - resultados de significância foram superiores a 0,05), quando da avaliação dos diferentes padrões de IMC por turma.

Fonte: Dados da pesquisa (2021).

No entanto, independente da turma, houve predominância significativa de eutrofia (ANOVA/Tukey p<0,05), em relação aos que se encontravam abaixo ou acima do peso (sobrepesos ou obesos) (Figura 1).

Figura 1 - IMC de alunos do curso de Nutrição do Centro Universitário UniFUNVIC. Pindamonhangaba/SP, Brasil. 2020.

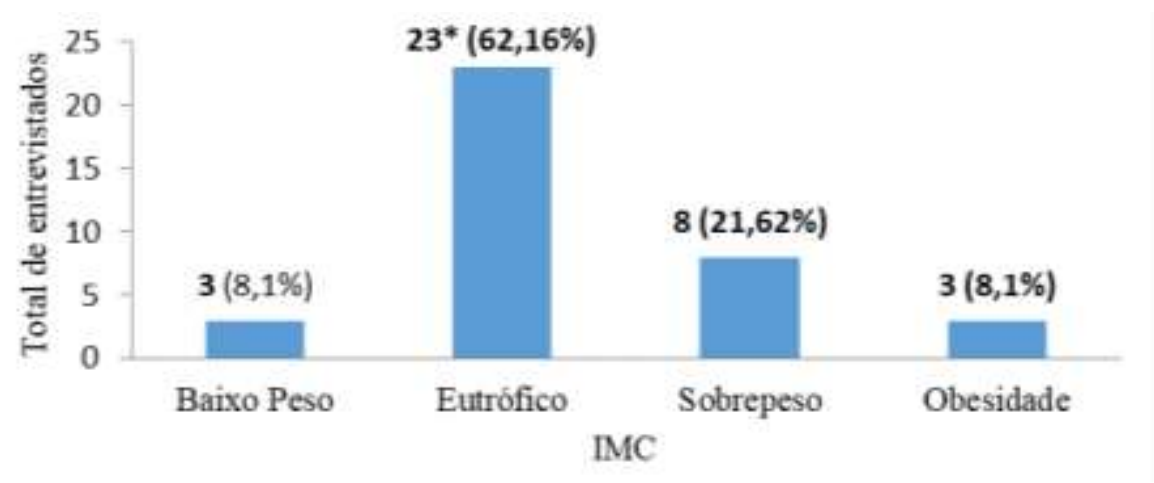

*diferença significativa em relação às demais categorias (ANOVA/Tukey, p<0,05) Fonte: Dados da pesquisa (2021).

\subsection{Consumo Alimentar}

Quando avaliada a influência do ingresso no ensino superior em nutrição no padrão de consumo alimentar, não houve diferença significativa entre as turmas avaliadas (teste G com significância <0,05), conforme observado no Quadro 6. Porém, de um modo geral, entre o total de alunos que participaram da pesquisa a maioria relatou ter mudado o padrão de 
consumo de alimentos após iniciar o curso, havendo diferença significativa (ANOVA/Tukey p<0,05) em relação aos que responderam o contrário.

Quadro 6 - Mudança no padrão de consumo de alimentos entre alunos do curso de Nutrição do Centro Universitário UniFUNVIC, Pindamonhangaba/SP, Brasil. 2020. Distribuição por turma.

\begin{tabular}{|l|c|c|}
\hline $\begin{array}{l}\text { Alimentação mudou ao iniciar a } \\
\text { Faculdade }\end{array}$ & Sim & Não \\
\hline $1^{\circ}$ ANO & $85.70 \%(n=6)$ & $14.30 \%(n=1)$ \\
\hline $2^{\circ}$ ANO & $66.70 \%(n=4)$ & $33.30 \%(n=2)$ \\
\hline $3^{\circ}$ ANO & $63.60 \%(n=7)$ & $36.40 \%(n=4)$ \\
\hline $4^{\circ}$ ANO & $84.60 \%(n=11)$ & $15.40 \%(n=2)$ \\
\hline Todos os anos & $75,67 \%(n=28)^{* *}$ & $24,32 \%(n=9)$ \\
\hline
\end{tabular}

*Ausência de diferença significativa (Teste G - resultados de significância foram superiores a $0,05)$, quando da avaliação dos diferentes padrões de IMC por turma

** Diferença significativa (ANOVA/Tukey, $\mathrm{p}<0,05$ )

Fonte: Dados da pesquisa (2021).

No que diz respeito à frequência de consumo de alimentos saudáveis (frutas, verduras e legumes) entre os alunos do curso de Nutrição do UniFUNVIC, pôde-se observar que, de um modo geral, os alunos optaram pelo consumo diário destes alimentos, sendo este hábito significativamente superior aos resultados relacionados às opções "nunca" ou "raramente" (Kruskal Wallis/Dunn p<0,05), conforme exposto na Figura 2.

Figura 2 - Frequência de consumo de alimentos saudáveis pelos alunos do curso de Nutrição do Centro Universitário UniFUNVIC. Pindamonhangaba/SP, Brasil. 202.

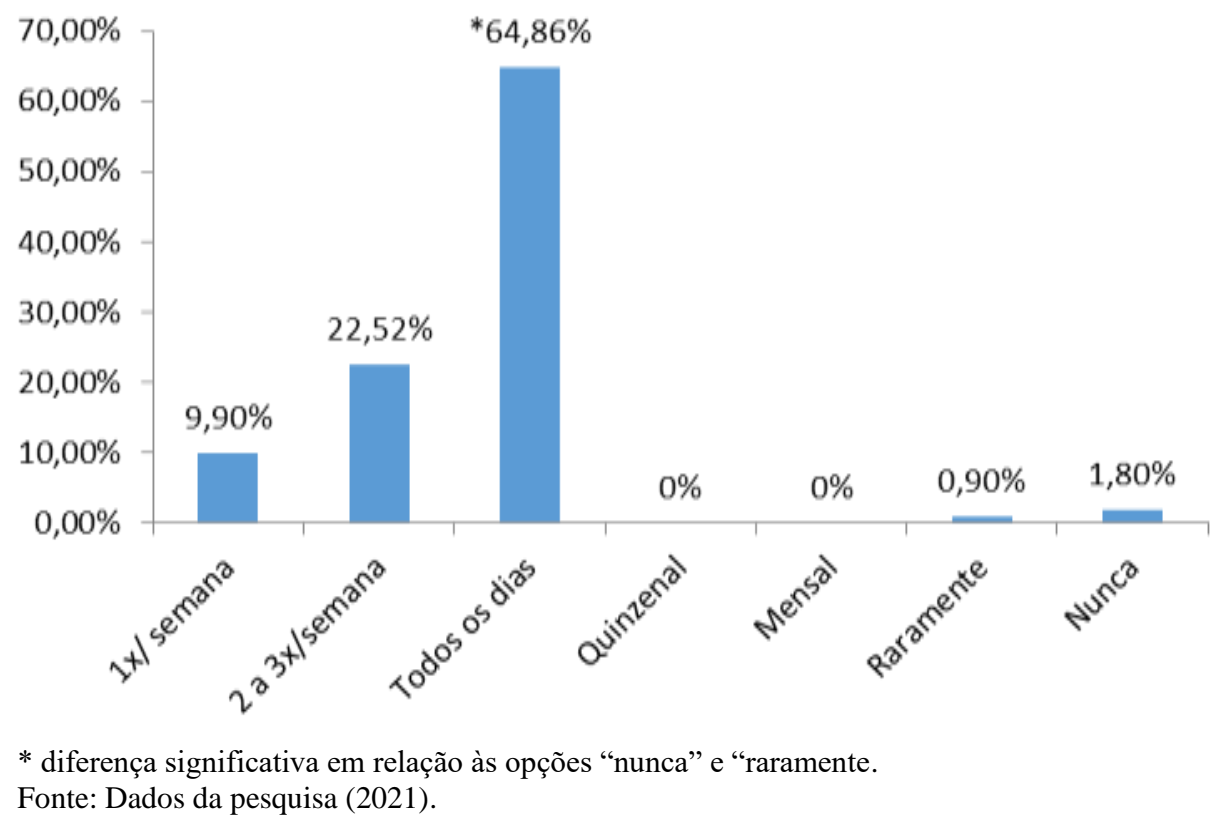

No entanto, no que diz respeito à frequência de consumo dos alimentos saudáveis por turma, avaliando frutas, verduras e legumes de forma geral e por uma mesma frequência, não foi observada diferença significativa após análise estatística (teste G, com significância <0,05) no consumo destes alimentos (Figura 3). 
Figura 3 - Frequência de consumo de alimentos saudáveis por parte de alunos do curso de Nutrição do Centro Universitário UniFUNVIC, após ingresso na Faculdade. UniFUNVIC. Pindamonhangaba/SP, Brasil. 2020

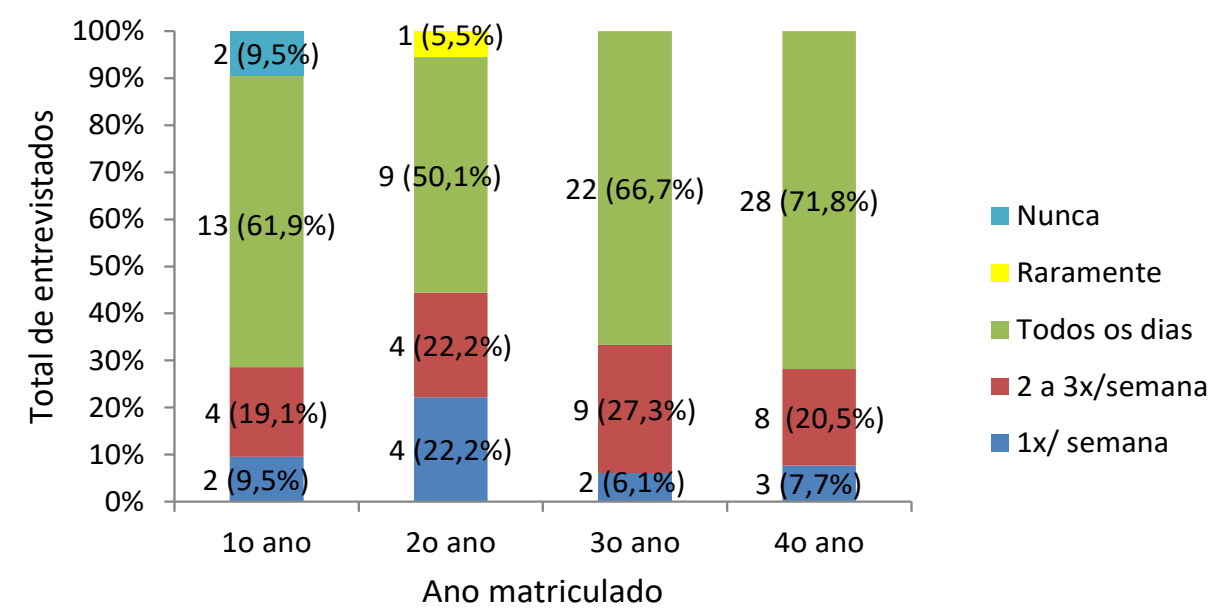

\footnotetext{
*Ausência de diferença significativa (Teste $\mathrm{G}$ - resultados de significância foram superiores a 0,05$)$, quando da frequência de consumo de alimentos saudáveis, por turma. Fonte: Dados da pesquisa (2021).
}

\subsection{Eating Attitudes Test -EAT-26}

Os resultados relacionados à ocorrência de transtornos alimentares no decorrer da graduação analisados pelo questionário EAT-26 estão descritos no Quadro 7. Após análises estatísticas (teste G, com significância <0,05 e teste binomial de duas proporções), observou-se não haver diferença significativa ao se comparar os índices de transtorno alimentar, observados nas diferentes turmas. Cabe destacar que ao se avaliar o total de respostas obtidas, independente da turma, houve predominância de ausência de transtorno, sendo esta significativa (Kruskal-Wallis/Dunn p<0,05), conforme observado na Figura 4.

Quadro 7 - Ocorrência de EAT em alunos do curso de Nutrição do Centro Universitário UniFUNVIC. .Pindamonhangaba/SP, Brasil. 2020. Distribuição por turma.

\begin{tabular}{|c|c|c|c|c|}
\hline EAT* & $\begin{array}{l}\text { Ausência } \\
\text { Transtorno }\end{array}$ & Transtorno Leve & $\begin{array}{l}\text { Transtorno } \\
\text { Moderado }\end{array}$ & Transtorno Grave \\
\hline $1^{\circ} \mathrm{ANO}$ & $85,70 \%(n=6)$ & $14,30 \%(\mathrm{n}=1)$ & $(n=0)$ & $(n=0)$ \\
\hline $2^{\circ} \mathrm{ANO}$ & $66,70 \%(n=4)$ & $33,30 \%(n=2)$ & $(\mathrm{n}=0)$ & $(\mathrm{n}=0)$ \\
\hline $3^{\circ} \mathrm{ANO}$ & $72,70 \%(n=8)$ & $18,20 \%(\mathrm{n}=2)$ & $9,10 \%(\mathrm{n}=1)$ & $(n=0)$ \\
\hline $4^{\circ} \mathrm{ANO}$ & $69,20 \%(n=9)$ & $30,80 \%(n=4)$ & $(n=0)$ & $(n=0)$ \\
\hline
\end{tabular}

*Não houve diferença significativa entre as turmas avaliadas (teste G - resultados de significância foram superiores a 0,05$)$.

Fonte: Dados da pesquisa (2021) 
Figura 4 - Ocorrência de EAT em alunos do curso de Nutrição do Centro Universitário UniFUNVIC. Pindamonhangaba/SP, Brasil. 2020.

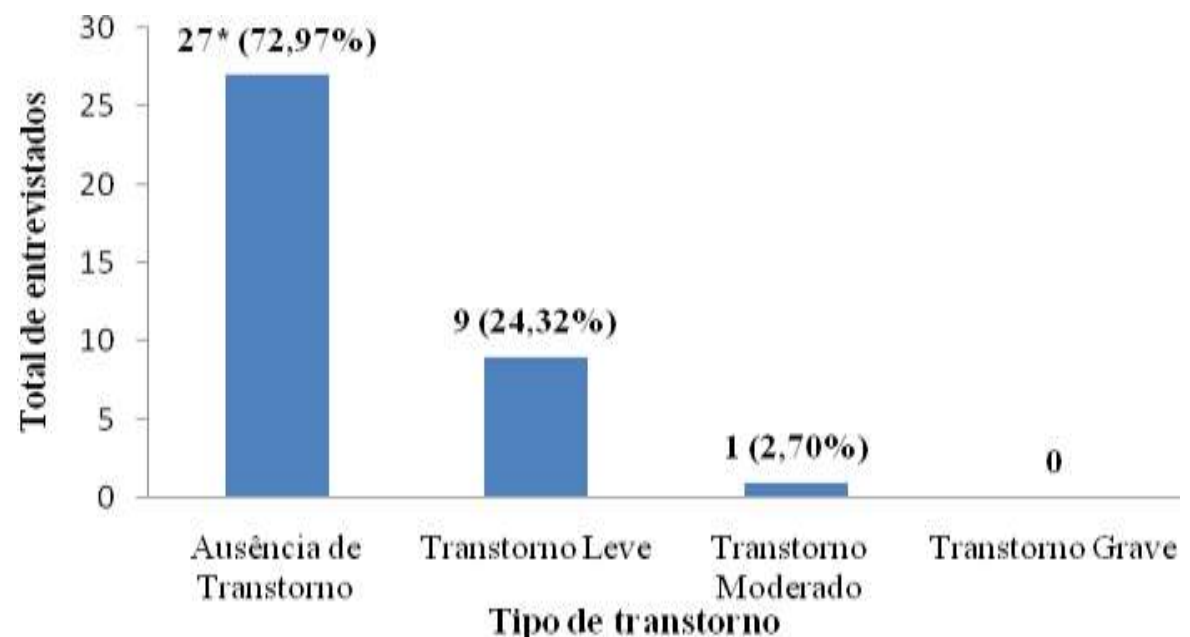

* Diferença significativa em relação às categorias: "Transtorno moderado" e "transtorno grave" (Kruskal

Wallis/Dunn, $\mathrm{p}<0,05)$

Fonte: Dados da pesquisa (2021).

\subsection{Body Shape Questionnaire - BSQ}

Por fim, ao avaliar possíveis distorções de imagem corporal entre os alunos das diferentes turmas do curso de nutrição pelo questionário BSQ, apesar de níveis de distorção moderada e grave estarem presentes apenas nas turmas do $6^{\circ}$ e $8^{\circ}$ períodos, após análise estatística (teste $\mathrm{G}$, com significância $<0,05$ ) não foi encontrada diferença significativa entre as turmas avaliadas (Quadro 8), porém, houve predominância significativa (ANOVA/Tukey, p<0,05) da ausência de distorção da imagem corporal em todas as turmas (Figura 5).

Quadro 8 - BSQ de alunos do curso de Nutrição do Centro Universitário UniFUNVIC. Pindamonhangaba/SP, Brasil, 2020. Distribuição por turma.

\begin{tabular}{|l|c|c|c|c|}
\hline BSQ* & $\begin{array}{c}\text { Ausência de } \\
\text { Distorção }\end{array}$ & $\begin{array}{c}\text { Distorção } \\
\text { Leve }\end{array}$ & $\begin{array}{c}\text { Distorção } \\
\text { Moderada }\end{array}$ & Distorção Grave \\
\hline $1^{\circ}$ ANO & $71.40 \%(n=5)$ & $28.60 \%(n=2)$ & $(n=0)$ & $(n=0)$ \\
\hline $2^{\circ}$ ANO & $50.00 \%(n=3)$ & $50.00 \%(n=3)$ & $(\mathrm{n}=0)$ & $(\mathrm{n}=0)$ \\
\hline $3^{\circ}$ ANO & $45.50 \%(\mathrm{n}=5)$ & $27.30 \%(\mathrm{n}=3)$ & $9.10 \%(\mathrm{n}=1)$ & $18.20 \%(\mathrm{n}=2)$ \\
\hline $4^{\circ}$ ANO & $69.20 \%(\mathrm{n}=9)$ & $(\mathrm{n}=0)$ & $7.70 \%(\mathrm{n}=1)$ & $23.10 \%(\mathrm{n}=3)$ \\
\hline
\end{tabular}

*Não houve diferença significativa entre as turmas avaliadas (teste $\mathrm{G}$ - resultados de significância foram superiores a 0,0001 )

Fonte: Dados da pesquisa (2021). 
Figura 5 - Percepção de distorção corporal em alunos do curso de Nutrição do Centro Universitário UniFUNVIC. Pindamonhangaba/SP, Brasil, 2020.

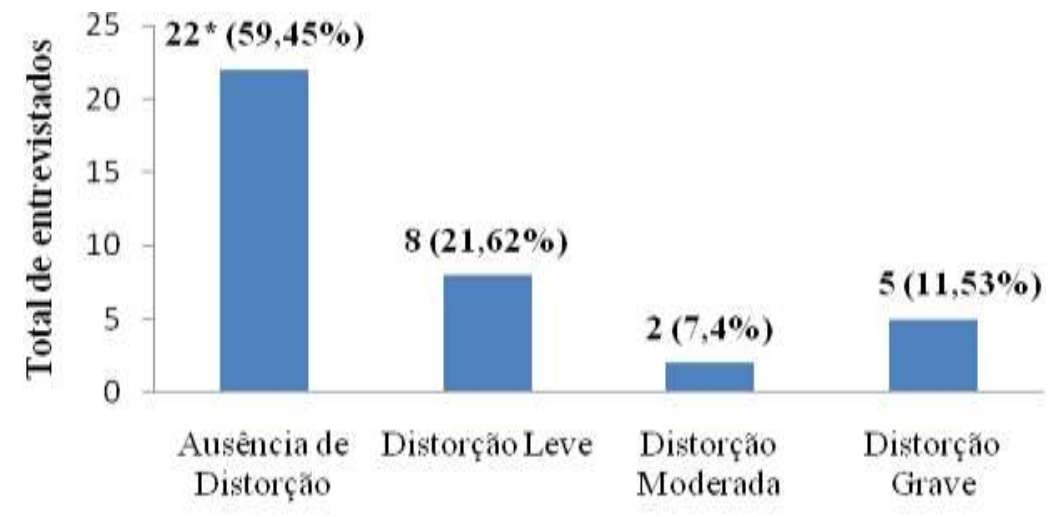

Intensidade de distorçâo

* diferença significativa em relação às demais categorias (ANOVA/Tukey, p<0,05) Fonte: Dados da pesquisa (2021).

Após análise da distorção de imagem, os resultados referentes à presença de desconforto com a aparência também foram analisados e estão demonstrados no Quadro 9. As análises estatísticas (teste G, com significância <0,05), demonstraram não haver diferença entre as turmas do curso de Nutrição ao se comparar a presença ou não de desconforto com a aparência. Também não foi observada diferença significativa (ANOVA/Tukey, com significância de 0,05) entre todos os alunos que responderam sentir ou não sentir algum desconforto com sua aparência.

Quadro 9 - "Desconforto com a aparência" de alunos do curso de Nutrição do Centro Universitário UniFUNVIC. Pindamonhangaba/SP, Brasil, 2020. Distribuição por turma.

\begin{tabular}{|l|l|l|}
\hline Desconfortável com a aparência* & Sim & Não \\
\hline $1^{\circ}$ ANO & $28.60 \%(n=2)$ & $71.40 \%(n=5)$ \\
\hline $2^{\circ}$ ANO & $33.30 \%(n=2)$ & $66.70 \%(n=4)$ \\
\hline $3^{\circ}$ ANO & $54.50 \%(n=6)$ & $45.50 \%(n=5)$ \\
\hline $4^{\circ}$ ANO & $38.50 \%(n=5)$ & $61.50 \%(n=8)$ \\
\hline Todos os anos** & $41,00 \%(n=15)$ & $59,00 \%(n=22)$ \\
\hline
\end{tabular}

*Não houve diferença significativa entre as turmas avaliadas (teste G - resultados de significância foram superiores a 0,05 ).

**Não houve diferença significativa nos dois scores avaliados (ANOVA/Tukey, significância foi superior a 0,05 )

Fonte: Dados da pesquisa (2021).

\section{Discussão}

No presente estudo, pode-se observar predominância do gênero feminino e faixa etária predominante entre 18 e 25 anos, o que corroborou com outros estudos, como o de Bernardino et al. (2019), que avaliaram a insatisfação da imagem corporal e possíveis transtornos alimentares em estudantes das áreas da saúde de uma faculdade particular de Bauru-SP e encontraram $70 \%$ da amostra composta por mulheres. Nascimento et al. (2020) estudaram 271 acadêmicos da área da saúde matriculados em uma universidade pública de Pernambuco e observaram que sua amostra foi composta por $62 \%$ de mulheres, com idade média de 21,2 anos. Bento et al. (2016) realizaram uma pesquisa transversal com uma amostra de 174 indivíduos, 
predominantemente feminina, onde a faixa etária predominante foi de 18 a 26 anos dos cursos de Enfermagem, Fisioterapia e Nutrição de uma Universidade de Petrolina/PE.

Quanto à prática de atividade física, notou-se que ao passar dos anos houve um aumento no percentual de alunos que praticavam alguma atividade física, uma vez que mais de $60 \%$ dos acadêmicos do último ano do curso de nutrição afirmaram praticar algum exercício, contra cerca de $43 \%$ dos concluintes. Na verdade, o percentual encontrado para o $4^{\circ}$ ano do curso foi acima do esperado e descrito em outros trabalhos na literatura. Santos et al. (2014) estudaram a qualidade de vida e alimentação de universitários de diferentes áreas em uma universidade de São Paulo e encontraram mais de 50\% dos indivíduos sedentários e Munhoz et al. (2017) estudando os hábitos alimentares e estilo de vida de 68 universitários de ambos os sexos, matriculados no curso de Nutrição de uma instituição privada de ensino superior em Araçatuba/SP, também encontraram que 68\% da amostra era sedentária. Ainett, Costa \& de Sá (2017) estudaram 133 estudantes de nutrição de uma Universidade Federal do Pará e encontraram apenas 6\% destes fisicamente ativos e dos Santos et al. (2021), estudando comportamento alimentar e imagem corporal de 1570 universitários da área da saúde, observaram que mais da metade dos acadêmicos $(53,4 \%)$ eram sedentários. Apenas Leite et al. (2011), em estudo realizado há mais tempo que os descritos anteriormente, encontraram resultados semelhantes aos do presente trabalho, observando um alto percentual de praticantes de atividade física $(66,9 \%)$ em acadêmicos de nutrição de uma instituição de ensino superior privada no estado de Santa Catarina e Pires et al. (2013), apesar de observarem que $87,7 \%$ das acadêmicas de um curso de enfermagem não realizavam qualquer atividade física, também encontraram maior prevalência de sedentarismo e inatividade física nos ingressantes, quando comparados aos alunos concluintes.

As razões para a maior prevalência de sedentarismo entre os ingressantes podem ser devidas às significativas mudanças em sua rotina, quando ficam expostos a uma sobrecarga de atividades disciplinares dentro e fora da sala de aula, passando várias horas sentados e com pouco tempo para alimentar-se e continuar ou iniciar um programa de atividade física (Pires et al., 2013), além da falta de tempo e recursos financeiros (Aquino, Pereira \& Reis, 2015; de Souza e Silva et al., 2021).

Bons níveis de atividade física são benéficos e suficientes para determinação e manutenção do estado de saúde e da condição de vida dos indivíduos (Del Duca et al., 2015). A liberação de endorfina na corrente sanguínea leva a um estado de euforia, redução da ansiedade, tensão e raiva, regulando, assim a função emocional. Neste sentido, a prática de atividade física pode contribuir para melhorar o estado emocional e prevenir comportamentos alimentares disfuncionais (Branco et al., 2012; Pitanga, Beck \& Pitanga, 2020).

No entanto, deve-se considerar outra provável explicação para a maior prevalência na prática de atividade física encontrada no presente estudo, em comparação a outros realizados com acadêmicos, é que a presente amostra foi composta apenas por estudantes de nutrição, cujo risco de perturbações do comportamento alimentar é superior, comparado aos estudantes de outros cursos, devido à pressão social para manutenção da aparência corporal dentro dos padrões exigidos pela sociedade, sendo também um forte grupo de risco para o desenvolvimento de transtornos alimentares (Bandeira et al., 2016; de Souza e Silva et al., 2021). Como os transtornos alimentares são multifatoriais, incluindo a ingestão alimentar e a prática de atividade física, nesse caso, a alta prevalência de atividade física observada neste estudo, pode estar desvinculada dos efeitos benéficos dos exercícios físicos sobre a saúde, mas relacionada exclusivamente ao desejo de perder peso (Vargas et al., 2016). Quando questionados sobre a presença de ansiedade e estresse, percebeu-se que a prevalência de ansiedade, de maneira geral, apresentou-se bastante presente nos acadêmicos de nutrição, com uma prevalência maior que $70 \%$, em todos os períodos avaliados. Ferreira, Silva \& Costa (2019) avaliaram o nível de ansiedade em estudantes da área da saúde em uma Universidade privada da Zona da Mata mineira e encontraram que os alunos dos cursos de enfermagem e nutrição foram os que mais relataram sintomas de ansiedade, mas a presença de ansiedade foi relatada em todos os cursos da área de saúde pesquisados, uns com menores e outros com índices altos e preocupantes, deixando claro que o meio acadêmico é extremamente vulnerável 
ao desenvolvimento de comportamentos ansiosos, seja pelo processo de integração do sujeito com o ambiente universitário, seja pelas transformações naturais da juventude, além de profundas mudanças de ordem econômica, social e cultural, que exigem grandes esforços do acadêmico para que a adaptação ao novo estilo de vida aconteça.

O nível de estresse observado no presente estudo foi aumentando, conforme o período em que se encontrava o aluno, estando presente em mais de $75 \%$ dos alunos concluintes. No entanto, não houve diferença significativa entre os períodos avaliados e, de maneira geral, percebeu-se que o estresse esteve predominante em todos os períodos, o que já era esperado, uma vez que é sabido que os universitários estão particularmente propensos ao estresse (Penaforte, Matta \&Japur, 2016). A revisão feita por Graner \& Cerqueira (2019) sobre sofrimento psíquico em estudantes universitários e fatores associados, apontou que os estudantes que mais manifestam tais sofrimentos psicológicos, são dos primeiros e últimos períodos do curso, explicado pelo fato de nos primeiros períodos o estudante estar passando pelos momentos de habituação à nova rotina de vida $\mathrm{e}$ acadêmica, além da distância de casa, dos familiares e dos amigos; já nos últimos, o indivíduo se depara com os processos e avaliações finais do curso e a proximidade da entrada no mercado de trabalho.

Em relação à satisfação com seu peso corporal, todos os alunos do $2^{\circ}$ ano, disseram não estar satisfeitos com seu peso. No entanto, quando avaliados em relação ao seu IMC, constatou-se que, quase a mesma proporção (cerca de $64 \%$ dos alunos do $3^{\circ}$ ano e $83 \%$ dos alunos do $2^{\circ}$ ) foi classificada como eutrófica. Dessa forma, percebeu-se que houve um alto índice de insatisfação com a imagem corporal nestes alunos, provavelmente por terem tendência a subestimar o próprio peso corporal, o que pode ser um reflexo de influências de fatores sociais e psicológicos (Boutahar et al., 2019).

Com relação ao IMC mensurado, apesar de serem observados casos de baixo peso no $1^{\circ}$ ano, 1 caso de sobrepeso no $2^{\circ}$ e casos de sobrepeso e obesidade no $3^{\circ}$ e $4^{\circ}$ anos, não foi encontrada diferença significativa no estado nutricional das diferentes turmas, com predominância significativa de eutrofia em todo o curso (cerca de 62\%). Tal situação corrobora com outros trabalhos, que obtiveram resultados semelhantes ao deste estudo, como o de Souza e Silva et al. (2019) ao analisar estudantes de nutrição de uma universidade pública em Recife e encontrar 77,1\% das mulheres e 59,1\% dos homens apresentando IMC adequado, bem como Oliveira et al. (2021) que encontraram $65,2 \%$ de eutrofia entre estudantes matriculados nos cursos de Nutrição e Letras da Universidade federal de Pelotas.

Foi questionado sobre a mudança na alimentação após o início da faculdade, que poderia estar relacionada ao ganho de conhecimento com a evolução do curso, e observou-se que em todos os anos, de alguma forma, houve mudanças nos hábitos alimentares, mais evidenciada nos alunos ingressantes e concluintes, em relação aos demais. Mais uma vez, tais resultados podem ser justificados pela maior manifestação de sofrimentos emocionais e psicológicos em estudantes dos primeiros e últimos anos do curso, uma vez que os ingressantes estão em fase de adaptação à nova rotina acadêmica e demandas sociais, somadas à nova liberdade e responsabilidade pela escolha, aquisição e preparo dos alimentos, além do enfrentando da distância de casa, da família e amigos e os concluintes, enfrentando processos e avaliações referentes à conclusão do curso, como estágios, trabalhos de conclusão, relatórios e ansiedade para a entrada no mercado de trabalho (Penaforte, Matta \& Japur, 2016; Graner \& Cerqueira, 2019).

Segundo a frequência alimentar dos estudantes durante a graduação, não houve diferença no consumo de alimentos saudáveis entre os diferentes períodos avaliados, porém, a frequência diária destes alimentos foi predominante em todos os períodos.

De maneira geral, houve alteração na alimentação dos estudantes, após entrada na universidade, o que pode ser explicado pela preocupação dos futuros nutricionistas em manterem uma "boa aparência", considerada uma importante medida para seu sucesso profissional, além do maior conhecimento a respeito dos alimentos, que praticamente os induzem a manterem-se dentro dos padrões estéticos vigentes (Bandeira et al., 2016; Munhoz et al., 2017). 
A ausência de transtornos alimentares, de maneira geral avaliada pelo questionário EAT-26 foi predominante nos alunos de todos os períodos, sendo mais evidenciada nos alunos ingressantes; o que contrapõe um estudo avaliado, já que o mesmo, relata encontrar valores significantes de transtorno alimentar ultrapassando $20 \%$ do total avaliado, o que é preocupante, principalmente entre os profissionais de saúde, onde o risco de transtornos alimentares se torna ainda maior (Souza et al., 2020).

Da mesma forma, a ausência de imagem corporal avaliada pelo questionário BSQ foi predominante na turma avaliada em todos os períodos, onde cerca de $60 \%$ dos alunos apresentaram ausência de distorção da imagem corporal, sendo essa ausência mais evidenciada nos alunos do primeiro ano. Ainett, Costa e de Sá (2017) estudando a satisfação com a imagem corporal em estudantes de nutrição, encontraram insatisfação com a imagem corporal em 59\% das alunas e Bernardino et al. (2019) verificaram através do mesmo teste, prevalência de 30,6\% de insatisfação e distorção de imagem corporal, dentre estudantes da área da saúde, assim como Maia et al. (2018) observaram que 46,1\% dos estudantes do curso de Nutrição do Instituto Federal de Educação, Ciência e Tecnologia do Ceará, apresentaram algum grau de insatisfação corporal. Tais resultados foram bastantes semelhantes aos observados no presente estudo, que é bastante preocupante considerando que cerca de $40 \%$ dos alunos avaliados possui algum tipo de transtorno alimentar e distorção de sua imagem corporal.

É sabido que alunos com distorção de imagem apresentam maior risco de transtornos alimentares. No entanto, entre os alunos com sobrepeso e obesidade, que relatam insatisfação com o peso, a distorção de imagem e transtornos alimentares, são mais comuns (Maia et al., 2018).

Estudos destacam que indivíduos que se preocupam com o controle de peso e autoimagem, optam em cursar nutrição, fato que também possa justificar o elevado risco para TA apresentados nessa população (Bandeira et al., 2016; de Souza e Silva et al, 2021).

\section{Conclusão}

Não foi observada influência significativa dos conhecimentos adquiridos durante o curso de nutrição pelo grupo de alunos avaliado, no desencadeamento de transtornos alimentares e/ou distorção de imagem corporal. Porém, foi notável que os hábitos alimentares destes universitários, e consequentemente, seu estado nutricional, estavam adequados, fatos que podem ser influenciados pela aquisição de novos conhecimentos na área. No entanto, também foi preocupante o elevado risco para transtornos alimentares e distorção da imagem corporal em toda a amostra.

Apesar do número total de alunos matriculados no referido curso ser pequeno, a adesão à pesquisa foi ainda menor $\mathrm{e}$ portanto, ressalta-se a necessidade de mais investigações, com um número maior de participantes, de forma a compreender os possíveis motivos relacionados aos sintomas de ansiedade e distorção de imagem corporal, relatados por grande parte dos acadêmicos.

\section{Referências}

Agopyan, A., Kenger, E. B., Kermen, S., Ulker, M. T., Uzsoy, M. A., \& Yetgin, M. K. (2018). The relationship between orthorexia nervosa and body composition in female students of the nutrition and dietetics department. Eating and Weight Disorders - Studies on Anorexia, Bulimia and Obesity. 24, 257266.

Ainett, W. do S., de O., Costa, V. V. L., \& de Sá, N. N. B. (2017). Fatores associados à insatisfação com a imagem corporal em estudantes de nutrição. Revista Brasileira de Obesidade, Nutrição e Emagrecimento, 11(62), 75-78.

Aquino, J. K., Pereira, P., \& Reis, V. M. C. P. (2015) Hábito e consumo alimentar de estudantes do curso de nutrição das Faculdades de Montes Claros Minas Gerais. Revista Multitexto, 3(1), 82-88.

Bandeira, Y. E. R., Mendes, A. L. R. F., Cavalcante, A. C. M., \& Arruda, S. P. M. (2016) Avaliação da imagem corporal de estudantes do curso de Nutrição de um centro universitário particular de Fortaleza. J Bras Psiquiatr.,65(2),168-73. 
Bento, K. M., Andrade, K. N. D., Silva, E. I. G., Mendes, M. L. M., Omena, C. M. B. D., Carvalho, P. G. S. D., \& Schwingel, P. A. (2016). Transtornos alimentares, imagem corporal e estado nutricional em universitárias de Petrolina-PE. Revista Brasileira de Ciências da Saúde, 20 (3),197-202.

Bernardino, M. R., Souza, C. T., Francisqueti, F. V., \& Souza, D. T. (2019). Avaliação da Insatisfação da Imagem corporal e possíveis Transtornos Alimentares em estudantes das áreas da saúde de uma faculdade particular de Bauru-SP. Revista Brasileira de Obesidade, Nutrição e Emagrecimento, 13,888897.

Bigheti, F., Santos, C. B., Santos, J. E., \& Ribeiro, R. P. P. (2004). Tradução e avaliação do Eating Attitudes Test em adolescentes do sexo feminino de Ribeirão Preto, São Paulo. Jornal Brasileiro de Psiquiatria, 53(6), 339-346.

Bosi, M. L. M., Luiz, R. R., Morgado, C. M. C., Costa, M. L. S., \& Carvalho, R. J. (2006). Auto percepção da imagem corporal entre estudantes de nutrição: um estudo no município do Rio de Janeiro. Jornal Brasileiro de Psiquiatria, 2(55),108-113.

Boutahar, K., Chetoui, A., Kaoutar, K., Najimi, M., \& Chigr, F. (2019). Anthropometric status and body image perception among Morocca nuniversity students. Rev Epidemiol Sante Publique, 67(5), 311-317.

Branco, J. C., Jansen, K., Wiener, C., Lopez, M., Porto, M., \& Silva, R. (2012). Prevalência de inatividade física e fatores associados ao transtorno de humor em adultos jovens. Revista Brasileira de Ciência e Movimento, 20(4), 60-68.

Cooper, P. J., Taylor, M. J., Cooper, Z., \& Fairbum, C. G. (1987) The development and validation of the Body Shape Questionnaire. Int J Eat Disord, 6, 48594.

Dantas, R. R., \& Da Silva, G. A. P. (2019) O papel do ambiente obesogênico e dos estilos de vida parentais no comportamento alimentar infantil. Rev Paul Pediatr.,37(3), 363-371

De Souza e Silva, L. C., Menezes, D. da S. D., Tomiya, M. T. O., Diniz, A. da S., Maio, R., de Arruda, I. K. G., Cabral, P. C., \& de Lemos, M. C. C. (2021). Estado nutricional, estilo de vida e consumo alimentar de estudantes de Nutrição de uma universidade pública. Brazilian Journal of Development, 7(4), 3977439790 .

Del Duca, G. F., Garcia, L., Rezende, L., Mielke, G. I., \& Guerra, P. H. (2015) Inatividade física, comportamento sedentário e saúde. In E. R., de Souza, J. V. do Nascimento, E. S. de Azevedo, B. O. Pereira. Educação física, lazer e saúde: interfaces ao desenvolvimento humano, (pp. 253-276) Florianópolis: UDESC.

Dos Santos, M. M., de Moura, P. S., Flauzino, P. A., Alvarenga, M. dos S., Arruda, S. P. M., \& Carioca, A. A. F. Comportamento alimentar e imagem corporal em universitários da área da saúde. (2021). J Bras Psiquiatr,70(2),126-33.

Ferreira, B. C., Silva, S. M., \& Costa, B. V. (2019) Verificação de ansiedade em acadêmicos dos cursos de saúde de uma universidade privada da Zona da Mata mineira. Interdisciplinary Scientific Journal, 6(5),330-353.

Fortes, L. de S., Amaral, A. C. S., Almeida, S. de S., Conti, M. A. \& Ferreira, M. E. C. (2016) Qualidades Psicométricas do Eating Attitudes Test (EAT26) para Adolescentes Brasileiros do Sexo Masculino. Psicologia: Teoria e Pesquisa, 32(3), 1-7

Garner, D. M., \& Garfinkel, P. E. (1979). The Eating Attitudes Test: An index of the symptoms of Anorexia nervosa. Psycological Medicine, 9(2),273-279.

Garner, D. M., Olmsted, M. P., Bohr, Y., \& Garfinkel, P. A. (1982). The eating attitudes test: psychometric features and clinical correlations. Psychology Medicine, 12, 871-878.

Graner, K. M., \& Cerqueira, A. T. A. R. (2019). Revisão integrativa: sofrimento psíquico em estudantes universitários e fatores associados. Revista Ciência e Saúde Coletiva, 24(4),1326-1346.

Karakus, B., Hidiroglu, S., Keskin, N., \& Karavus, M. (2017). Orthorexia nervosa tendency among students of the department of nutrition and dietetics at a university in Istanbul. Northern clinics of Istanbul, 4(2), 117-123.

Leitão, P., Pimenta, F. Herédia, T., \& Leal, I. (2013). Comportamento alimentar, compulsão alimentar, história de peso e estilo de vida: diferenças entre pessoas com obesidade e com uma perda de peso bem-sucedida. Alim Nutr. = Braz J Food Nutr, 24(4), 393-401.

Leite, A. C. B., Grillo, L. P., Caleffi, F., Mariath, A. B., \& Stuker, H. (2011) Qualidade de vida e condições de saúde de acadêmicos de nutrição. Espaço para Saúde, 13(1), 82-90.

Maia, R. G. L., Fiorio, B. C., de Almeida, J. Z., \& da Silva, F. R. (2018). Estado nutricional e transtornos do comportamento alimentar em estudantes do curso de graduação em nutrição do Instituto Federal de Educação, Ciência e Tecnologia, Ceará, Brasil. Demetra: alimentação, nutrição \& saúde, 13(1),135-145.

Munhoz, M. P., Oliveira, J., dos Anjos, J. C., Gonçalves, R. D., Lopes, J. F., \& Celemi, L. G. Perfil nutricional e hábitos alimentares de universitários do curso de nutrição. (2017) Revista Saúde UniToledo, 1(2), 68-85.

Nascimento, V. S. D., Santos, A. V. D., Arruda, S. B., Silva, G. A. D., Cintra, J., Pinto, T. C. C., \& Ximenes, R. C. C. (2020) Associação entre transtornos alimentares, suicídio e sintomas depressivos em universitários de cursos de saúde. einstein (São Paulo),18, 1-7.

Oliveira C. N., Contreira, R. de L., de Vargas, L. B., Magalhães, L. S., Moreira, A. N., \& Pretto, A. D. B. (2021). Compulsão alimentar em universitários de uma cidade de médio porte do Rio Grande do Sul. BRASPEN J, 36 (1), 20-6

Oliveira, J. de S., Santos, D. de O., Rodrigues, S. J. M., de Oliveira, C. C., \& Souza, A. L. da C. (2018). Avaliação do perfil sociodemográfico, nutricional e alimentar de estudantes de nutrição de uma universidade pública em Lagarto-SE. Revista Da Associação Brasileira De Nutrição - RASBRAN, 8(2), 37-42.

Penaforte, F, R., Matta, N. C., \& Japur, C. C. (2016) Associação entre estresse e comportamento alimentar em estudantes universitários. Demetra: alimentação, nutrição \& saúde,11(1),225-237. 
Research, Society and Development, v. 10, n. 14, e248101421686, 2021

(CC BY 4.0) | ISSN 2525-3409 | DOI: http://dx.doi.org/10.33448/rsd-v10i14.21686

Pereira, C. M., da Silva, A. L. \& de Sá, M. I. (2015). Fatores que influenciam os comportamentos alimentares: questionário das escolhas alimentares dos adolescentes. Psicologia, Saúde \& Doenças, 16(3), 421-438.

Pires, C. G. D. S., Mussi, F. C., Cerqueira, B. B. D., Pitanga, F. J. G., \& Silva, D. O. D. (2013) Prática de atividade física entre estudantes de graduação em enfermagem. Acta Paulista de Enfermagem, 26(5),436-443.

Pitanga, F. J. G., Beck, C. C., \& Pitanga, C. P. S. (2020) Atividade Física e Redução do Comportamento Sedentário durante a Pandemia do Coronavírus. Arq Bras Cardiol. 114(6),1058-1060.

Santos, A. K. G. V., Reis, C. C., Chaud, D. M. A., \& Morimoto, J. M. (2014) Qualidade de vida e alimentação de estudantes universitários que moram na região central de São Paulo sem a presença dos pais ou responsáveis. Rev. Simbio-Logias, 7(10), 76-99.

Sousa, F. D. C. A., Oliveira, J. C. R., Alves, F. R., da Silva, W. C., De Sá Rodrigues, R. P., Da Silva, A. B. S., \& Mendes, R. C. (2020) Estudo do estado nutricional, imagem corporal e atitudes para transtornos alimentares em acadêmicas de nutrição. Revista Enfermagem Atual In Derme, 93(31),6-7.

Vargas, L. M., Cantorani, J. R. H., Mauricio, L. K., \& Annunciato, S. K. L. (2016). Nível de atividade física de estudantes universitários com e sem distúrbio da imagem corporal. Pensar a Prática, 19(1),169-181.

Yu, Z., \& Tan, M. (2016) Disordered Eating Behaviors and Food Addiction among Nutrition Major College Students. Nutrients, 8(11),673 